\title{
Commentary on High-Performance Computing and Psychological Models: Theory-building and model-fitting processes
}

\author{
STEPHEN W. LINK \\ Federation of Behavioral, Psychological and Cognitive Sciences, Washington, D.C.
}

\begin{abstract}
For a psychological theorist, a merging of the interactive capability of a desktop computer and the speed memory capability of a supercomputer is needed. Ready-to-use modeling software is especially useful in the behavioral sciences so that researchers do not need to reinvent each other's wheels. Dynamical models of psychological processes require different analyses from routine statistical treatments.
\end{abstract}

Shiffrin and Nobel (1997) discuss the application of supercomputers in testing psychological models of cognitive processes. Shiffrin points out that while a model's parameter space may be easily defined, the choice of the function measuring the "fit" between the model and data is arbitrary but crucial. The function chosen to determine the parameters yielding a "best fit" to data can generate an awkward-looking minimization space with local maxima and minima. Even with supercomputing speed, computing time limitations and a finite life-span limit the theorist seeking a "best fit."

Practically speaking, the theorist cannot investigate all points in the parameter space. Furthermore, the minimization function itself may not be equally weighted across the entire range of the data space-some observations may be so aberrant that deviations of predictions from these "outliers" should not determine the "best" set of model parameters. What is needed is the capability to interact with the program that fits the model to the data. Then the theorist can try new ideas quickly, thereby reducing the parameter space to optimal values based on an understanding of the complexities of the data and the particular minimization function. Somehow the interactiveness of the desktop computer needs to be tied to the speed of the supercomputer.

An attendant problem, as Suppes (1997) notes, is that there seems to be a lack of ready-to-use modeling software, especially in the behavioral sciences. As a consequence, those who fit models to data somehow end up repeating a great deal of what has already been explored. The reinvention of techniques for fitting the theories to data results, in part, from the lack of any consensus on a model. In the field of memory research, for example,

S. W. Link's mailing address is Department of Psychology, McMaster University, Hamilton, ON, Canada L8S 4K1 (e-mail: link@mcmaster.ca). there are four models that undergo extensive investigation. Shiffrin and his students wrote software for the Shiffrin model, Murdock's SAM, Humphrey's matrix model, and Hintzman's neural model. But in each case, the program must be tailored to the specific experimental conditions.

Providing a general model in the behavioral sciences is difficult because we have less confidence in our models than physicists do in their models. None of us seriously believes that the current model of, say, memory is sufficiently precise to explain the data. The goodness-offit statistics useful in physics may not differentiate good psychological models from bad, because of the amount of noise inherent in behavioral experiments and the variability of individuals who are the subjects in them.

Another aspect of the problem of determining goodness of fit using interactive computing is the method of deciding when one theory does a substantially better job than another. For example, no one really believes in linear regression either, but it provides a reasonable baseline for the purpose of comparing predictions. In order to present one model as superior, we need a baseline against which to compare it.

Shiffrin and Nobel (1997) note that there are two types of models. One is descriptive, as is the regression model. The other is a model of the dynamical processes that are the foundation for observed performance, as is the temporal Markov field model of text schemata proposed by Golden (1997). The latter type of model is not yet at the stage at which a baseline for performance can be established, in part because of the novelty of the statistical estimation problems posed and in part because there are no competing models that satisfactorily account for even the qualitative aspects of data.

What is becoming increasingly clear is that the models in psychology are as dynamic and compelling as models to be found in the physical sciences. But the structure 
of such models is quite different from those that gave rise to the statistical treatments often imported from the physical sciences into psychology. At this stage of theory development in psychology, additional theoretical and computational attention must be devoted to the estimation problems created by this new class of theories. Given the complexity of the problems, the on-line use of supercomputers can provide powerful assistance in the testing of these theories.

\section{REFERENCES}

GoLDEN, R. M. (1997). Causal network analysis validation using synthetic recall protocols. Behavior Research Methods, Instruments, \& Computers, 29, 15-24.

SHIFFIN, R. M., \& NoBEL, P. A. (1997). The art of model development and testing. Behavior Research Methods, Instruments, \& Computers, 29, 6-14.

SUPPES, P. (1997). Perception, models, and data: Some comments Behavior Research Methods, Instruments, \& Computers, 29, 109 112. 